\title{
Perspectiva tecnológica de la práctica contable. Ontología y episteme de la contabilidad de gestión *
}

\author{
Samuel Leonidas Pérez-Grau \\ Docente Universidad Simon Bolivar, Barranquilla - Colombia \\ sammy975603@gmail.com (D) https://orcid.org/0000-0002-4105-0819
}

RES U ME N

Este estudio pretende establecer una fundamentación ontológica y epistemológica que confiera a la Contabilidad de Gestión "razón de ser" como una tecnología científica contable unificadora de diversos elementos del ejercicio profesional contable. La existencia de diversas líneas tecnológicas como contabilidad administrativa, contabilidad de costos o contabilidad analítica, a veces cambian la visión de la ciencia de la contabilidad dando a entender que éstas fuesen ápice de una disciplina diferente. Este trabajo es fruto de una reflexión teórica sustentada en diversas elaboraciones sobre contabilidad de gestión y en diferentes documentos bibliográficos relacionados con declaraciones de principios y marcos conceptuales que preparan organizaciones gremiales internacionales de contadores de gestión. Entre los hallazgos más importantes se destaca que la contabilidad de gestión es una auténtica praxis social con gran potencial de méritos para posicionarse como tecnología contable.

Palabras clave Contabilidad de gestión, eje transversal, modelización, simulación, innovación digital CLASIFICACIÓN JEL M4I, M2I, MI6

\section{Technological perspective of accounting practice. Ontology and episteme of management accountin}

A B S T R A C T

This study intends to establish an ontological and epistemological foundation that confers on Management Accounting "raison d'être" as a unifying scientific accounting technology of various elements of professional accounting practice. The existence of different technological lines such as administrative accounting, cost accounting or analytical accounting, sometimes change the vision of accounting science, implying that these were the apex of a different discipline. This work is the result of a theoretical reflection based on various elaborations on management accounting and in different bibliographic documents related to statements of principles and conceptual frameworks prepared by international trade organizations of management accountants. Among the most important findings is that management accounting is an authentic social praxis with great merit potential to position itself as an accounting technology.

KEYWORDS

Management accounting, transversal axis, modeling, simulation, digital innovation

JEL CLASSIFICATION

M4I, M2I, MI6

Recibido: 10/05/2019 Aceptado: 15/06/2019

\footnotetext{
* Este artículo es un producto del proyecto de investigación denominado Determinación del quehacer profesional del contador público en contabilidad de gestión, desarrollado por el grupo Pensamiento Contable y Negocios Internacionales de la Universidad Simón Bolívar, Barranquilla, Colombia, financiado con recursos institucionales y propios del investigador

http://dx.doi.org//0.18041/1900-3803/entramado.2.5649 Este es un artículo Open Access bajo la licencia BY-NC-SA

(http://creativecommons.org/licenses/by-nc-sa/4.0/) Publicado por Universidad Libre - Cali, Colombia.
}

Cómo citar este artículo: PÉREZ-GRAU, Samuel Leonidas. Perspectiva tecnológica de la práctica contable. Ontología y episteme de la contabilidad de gestión. En: Entramado. Julio - Diciembre, 2019. vol. 15, no. 2, p. I20-128. http://dx.doi.org//0.18041//900-3803/entramado.2.5649 


\section{Perspectiva tecnológica da prática contábil. Ontologia e episteme da contabilidade gerencial}

Este estudo pretende estabelecer uma fundamentação ontológica e epistemológica que confira a "razão de ser" da Contabilidade Gerencial como uma tecnologia de contabilidade científica unificadora de vários elementos da prática contábil profissional. A existência de várias linhas tecnológicas, como contabilidade administrativa, contabilidade de custos ou contabilidade analítica, às vezes muda a visão da ciência contábil, implicando que estes eram o ápice de uma disciplina diferente. Este trabalho é resultado de uma reflexão teórica baseada em várias elaborações sobre contabilidade gerencial e em diferentes documentos bibliográficos relacionados a declarações de princípios e marcos conceituais elaborados por organizações internacionais de comércio de contadores gerenciais. Entre os achados mais importantes, destaca-se que a contabilidade gerencial é uma autêntica práxis social com grande potencial de mérito para posicionar-se como tecnologia contábil.

Palavras-chave Contabilidade de gestão, eixo transversal, modelagem, simulação, inovação digital Classificações JEL M4I, M2I, MI6

\section{Introducción}

El diseño metodológico de este artículo adopta un enfoque descriptivo y reflexivo que expresa sus resultados desde una perspectiva analítica, interpretativa y crítica del autor, a partir de una revisión documental sobre avances disciplinarios de la Contabilidad de Gestión (CG).

La CG es una rama de la contabilidad que tiene por objeto la captación, medición y valoración de la circulación interna, así como su racionalización y control, con el fin de suministrar a la organización, la información relevante para la toma de decisiones empresariales (AECA, 2003, p. 23).

En el estado actual del conocimiento, la CG es considerada como la activadora del sistema de información de las organizaciones y los contadores de gestión, son vistos como los creadores de valor de las operaciones y los procesos.

Dada la necesidad de información detallada sobre el estado presente de las operaciones, los reportes de la CG son típicamente más específicos que los informes tradicionales de la contabilidad financiera. Muchas compañías ahora esperan que su personal de CG participe en equipos interfuncionales con otros gerentes en toda la organización y ayude a desarrollar estrategias para mejorar la riqueza de los accionistas.

Para facilitar este papel cada vez más interactivo, algunos observadores de la profesión creen que los nuevos profesionales contables necesitarán una base empresarial más amplia en sus antecedentes académicos y profesionales.

La organización del artículo comienza con la introducción, seguida del desarrollo, éste en parcelas sobre historia, teoría y metodología de la Contabilidad de Gestión, para llegar a las conclusiones. El documento termina con la compilación de las referencias bibliográficas originales.

\section{Desarrollo}

Es a partir de 1980 cuando surge entre las asociaciones de profesionales el interés para abordar los problemas que empezaba a enfrentar la práctica contable para apropiar las nuevas habilidades exigidas por la introducción profunda de la sistematización informática que forzaba a los contadores a adoptar un nuevo rol ocupacional como asesores gerenciales en los procesos de toma de decisiones.

La primera declaración sobre Contabilidad de Gestión (CG) estuvo referida a costos gerenciales y fue presentado al público en Julio de 2012, como un borrador de exposición en el que el Instituto de Contadores de Gestión (IMA, 2014, p. 128), conocido anteriormente como la Asociación Nacional de Contadores (NAA por sus siglas en inglés) solicitaba comentarios a los practicantes y usuarios contables de gestión. Posteriormente, el primer conjunto de Principios de Contabilidad de Gestión (PMA por sus siglas en inglés) puesto a disposición de los practicantes surge en 2014 (King, 20I5, p.I), presentada conjuntamente entre el Instituto de Contadores de Gestión Certificados (ICMA por sus siglas en inglés) y el Instituto Americano de Contadores Públicos Certificados (AICPA por sus siglas en inglés).

En 2017, tiene lugar la emisión del primer marco conceptual sobre CG, presentado por la unión entre el Instituto Americano de Contadores Públicos Certificados (AICPA) y el Instituto de Contadores de Gestión Certificados (ICMA), bajo el título de Los Principios Globales de Contabilidad de Gestión (CIMA-AICPA, 20I7, p. 3), emitidos como una guía global de Buenas Prácticas de CG para Contadores de Ges- 
tión Mundialmente Certificados (CGMA). Ellos reflejan la perspectiva de los gerentes funcionales (CEOs y CFOs), los académicos y otros profesionales que contribuyeron durante una consulta global en los cinco continentes.

En este lapso, han sido numerosos los estudios de investigación hechos en todo el mundo para detectar tanto el fortalecimiento de la CG y la inclusión de nuevos temas, como la estructura y evolución de este campo del conocimiento científico. Los trabajos más conocidos son, uno, el realizado por Hesford y Potter (2010) analizando 916 artículos publicados durante 20 años (198I-1990 y I99|-2000) en 10 revistas de contabilidad en lengua anglosajona, incluidas en el Social Science Citation Index (SSCl) y, el otro, realizado por Lunkes, Ripoll y Silva (20I2) analizando 42I artículos de 7 revistas españolas registradas en INRECS y $32 \mathrm{I}$ artículos de 7 revistas brasileñas registradas en Publindex, durante 200 I-20 I 0. Los resultados muestran que los trabajos en CG propiamente dicha no han ocupado un lugar destacado en las publicaciones analizadas.

La Tabla I muestra un resumen de la composición y frecuencia de los temas afines a este estudio, tomado de dichos trabajos.

De lo anterior, puede colegirse que las publicaciones investigadas son diversas y además distintas, centrando por ello la clasificación en las secciones temáticas más incluyentes de costes, planificación y control, y planeación estratégica. Sobre costes, lo que más se publica es lo conocido en nuestro medio como contabilidad de costos (58\%), en cuanto a planificación y control las mayores publicaciones son sobre control interno (37\%), control de gestión (25\%) y presupuestos (18\%), mientras que sobre gestión estratégica más se publica sobre sistema de información (20\%) y educación en gestión (15\%).

En materia de la praxis laboral, mencionan Pérez-Grau y Zabaleta (2017, p. 109) que:"la tendencia dominante en CG desde la última mitad del siglo anterior ha sido la del uso del dato contable interno crecientemente detallado para ayudar a timonear las decisiones gerenciales y mejorar los rendimientos operacionales". Generalmente, los profesionales al servicio de la organización encargados de la función de CG respaldan dos tipos de propósitos primarios.

El primero, se refiere a la generación de reportes de rutinas que contienen información sobre el control de costos y la planeación y el control de las operaciones. Esta información producida ayuda a los gerentes a incrementar el conocimiento de la organización acerca del futuro y a reducir los riesgos asociados con la toma de decisiones y acciones pasadas. Las ocupaciones principales que encontrará el con122 ponderán a los cargos de:

- Administrador del sistema computacional (Data gobernance, Blockchain, Control de riesgos)

- Gestor de bases de datos (Big data,Artificial intelligence, Previsive analytics)

El segundo, consiste en la producción de informes reglamentados de los contadores de gestión sobre materias relacionadas con el costo de producción, los gastos relacionados con los programas de capacitación, los costos del mercadeo, la asignación de precios a los productos o servicios, la escogencia de las líneas que deban ser enfatizados o no, inversiones en equipos y la formulación de políticas generales y planeación del largo plazo.Aquí, las ocupaciones principales corresponderán a los cargos de:

- Manejador (Controller) del software de procesos de negocio (Life cycle costing (LCC), Activities based costing (ABC), Block chain (BC), Lean Production (LP), Activities based budget (ABB), Balance Score Card (BSC).

- Digitador de contenidos Extensible Bussines Report Language (XBRL)

Es importante mencionar que, tanto para la elaboración de estos informes como para la valoración de los recursos, operaciones y procesos, los profesionales contables utilizan datos reales tomados directamente de los sistemas logísticos y operacionales en vez de las cuentas del libro mayor general, es decir, el profesional contable afronta la búsqueda y evaluación de alternativas como etapas previas a la elección final de una decisión.

Vale aquí mencionar que la CG además de proveer información a los administradores también ejerce evaluación, para ello sigue una nueva cualificación del resultado de la organización denominada "modelo de gestión" que incorpora tanto los resultados como las decisiones, para las distintas áreas de responsabilidad, unidades de negocios y complementa la evaluación del desempeño de sus administradores (Pérez-Grau, 2014).

La liga de lo tecnológico a lo científico contable, surge a partir de la observación racional del estado presente de la cadena de valor interna de la organización, seguida de la necesidad de su validación como información útil para terceros, quienes a partir de teorías disponibles como la teoría de los juegos, la teoría de la utilidad cardinal y la teoría de las restricciones, podrán garantizar a la sociedad la comprobación de la verdad del trabajo hecho por los contadores (Peñaloza, M.P. 20I0, p. 230).

Seguidamente, al incursionar acerca de los aspectos teóricos, se encuentra que las dos teorías que propician explica- 
Tabla I.

Comparación entre estudios de publicaciones sobre la contabilidad de gestión

\begin{tabular}{|c|c|c|c|c|}
\hline TEMAS & $\begin{array}{c}\text { SSCI } \\
1981-1990\end{array}$ & $\begin{array}{c}\text { SSCI } \\
1991-2000\end{array}$ & $\begin{array}{l}\text { IN-RECS } \\
2001-2010\end{array}$ & $\begin{array}{c}\text { PUBLINDEX } \\
\text { 2001-2010 }\end{array}$ \\
\hline \multicolumn{5}{|l|}{ COSTES } \\
\hline Contabilidad de costes & $36(11,3 \%)$ & $104(17,5 \%)$ & $43(10,5 \%)$ & $27(8,5 \%$ \\
\hline Gestión de costes & $14(4,4 \%)$ & $7(1,2 \%)$ & $7(1,5 \%)$ & $40(12,5 \%)$ \\
\hline Estudios de costes & $4(1,3 \%)$ & $11(0,2 \%)$ & $27(6,5 \%)$ & $7(2 \%)$ \\
\hline Gestión estratégica de costes & - & - & $14(3,5 \%)$ & - \\
\hline Otros & - & $1(0,2 \%)$ & $17(4 \%)$ & - \\
\hline Subtotal & $54(16,9 \%)$ & $123(20,6 \%)$ & $108(25,7)$ & $74(23 \%)$ \\
\hline \multicolumn{5}{|l|}{ PLANIFICACIÓN Y CONTROL } \\
\hline Presupuesto & $64(20 \%)$ & $70(11,7 \%)$ & $34(8 \%)$ & $10(3 \%)$ \\
\hline Presupuesto financiero & $14(4,4 \%)$ & $33(5,5 \%)$ & $5(1 \%)$ & $29(9 \%)$ \\
\hline Control de gestión & $35(10,9 \%)$ & $113(19 \%)$ & $55(13 \%)$ & $38(12 \%)$ \\
\hline Control interno & $119(37,2 \%)$ & $177(29,7 \%)$ & $21(5 \%)$ & $46(14,5 \%)$ \\
\hline JIT y TOC & - & $7(1,2 \%)$ & $2(0,5 \%)$ & $10(3 \%)$ \\
\hline Precios de transferencia & $9(2,8 \%)$ & $22(3,7 \%)$ & $17(4 \%)$ & $3(1 \%)$ \\
\hline Otros & $5(1,6 \%)$ & $14(2,3 \%)$ & 12 & 18 \\
\hline Subtotal & $246(77 \%)$ & $436(73,1 \%)$ & $146(34,7 \%)$ & $154(48 \%)$ \\
\hline \multicolumn{5}{|l|}{ GESTIÓN ESTRATÉGICA } \\
\hline Planeación estratégica & $7(2,2 \%)$ & $8(1,3 \%)$ & $12(3 \%)$ & $19(6 \%)$ \\
\hline Sistema de información & $4(1,3 \%)$ & $3(0,5 \%)$ & $26(6 \%)$ & $30(9,5 \%)$ \\
\hline Benchmarking & - & $2(0,3 \%)$ & $4(1 \%)$ & $1(0,5 \%)$ \\
\hline TQM & - & $9(1,5 \%)$ & $25(6 \%)$ & $3(1 \%)$ \\
\hline Educación en gestión & $6(1,9 \%)$ & $14(2,4 \%)$ & $4(1 \%)$ & $25(7 \%)$ \\
\hline Análisis financiero & - & - & $31(7,5 \%)$ & $3(1 \%)$ \\
\hline Estudios en CG & - & - & $28(6,5 \%)$ & $6(2 \%)$ \\
\hline Otros & $3(0,1 \%)$ & $1(0,2 \%)$ & $37(8,5 \%)$ & $6(2 \%)$ \\
\hline Subtotal & $20(6,3 \%)$ & $37(6,2)$ & $167(39,7 \%)$ & $93(29 \%)$ \\
\hline Total & $320(100 \%)$ & $596(100 \%)$ & $421(100 \%)$ & $321(100 \%)$ \\
\hline
\end{tabular}

Fuente: Lunkes, R., Ripoll,V.y Silva F. 20 I2, p. I 8)

ciones más viables sobre la manera de difundir la tecnología contable de la CG, en el medio profesional-empresarial, son: la teoría institucional y la teoría de las lógicas institucionales.

Desde una perspectiva institucional la adopción de una innovación digital pasa por dos etapas de investigación potencialmente fructíferas, la primera, la contingencia de adecuación de la organización entre los nuevos sistemas de administración, ambiente y tecnología y la segunda, la comprensión de la legitimidad que otorga el isomorfismo mimético, ya sea porque tal innovación está siendo difundida como el estándar profesional apropiado (legitimidad normativa) o porque ha sido legislada debido a su conveniencia social (legitimidad coercitiva) (Meyer y Rowan, 1977 y Williamson O.E., 2000).

Desde el punto de vista de la teoría de las lógicas institucionales (DiMaggio y Powell, 1983) es a través del vínculo entre los compromisos acordados (la agencia) y las prácticas socialmente construidas, que dan forma a los comportamientos conscientes con los que los actores individuales y organizacionales participan en la producción y reproducción de sus vidas materiales y hacen significativas sus experiencias. En intensidad, según que las instituciones sobresocialicen una intención de internalización y de valores o una intención insuficientemente socializada sobre manejo de recursos e intereses políticos (Thornton y Ocasio, 2012).

La innovación digital se refiere a la adopción concertada de nuevos productos, nuevos procesos, nuevos servicios, nuevas plataformas, o incluso nuevos modelos comerciales en un contexto dado (Nambisan, 2016). La transformación digital se trata de la acción orquestada de varias innovaciones digitales sobre estructuras, prácticas, valores y creencias, actores noveles (y constelaciones de actores), que comple- 
menta, reemplaza, amenaza, o cambia, las reglas existentes del juego dentro de las organizaciones, ecosistemas, industrias o campos (Loebbecke y Picot, 2015).

En el estado actual de desarrollo tecnológico (Hinings, Gegenhuber y Greenwood, 2018), los tipos principales de arreglos institucionales, digitalmente transformados o habilitados por acción de la CG, son:

- Instituciones digitales en forma de bloques de construcción: Estas se componen de elementos modulares generalmente aceptados, listos y personalizables, como el ERP, que abarcan conjuntos de tecnologías digitales para ejecutar o crear una organización (Nambisan, 2016). En ellas, dos cosas se destacan. En primer lugar, se plantean como verdaderamente transformadoras debido a la posibilidad de establecer diversos patrones innovadores. Segundo, como toda innovación y cambio dentro de un argumento institucional, cada bloque de construcción debe estar dotado de una legitimidad separada al tiempo que cualquier nuevo arreglo institucional surgido debe gozar de una legitimidad colectiva.

- Infraestructuras institucionales digitales: Estas son innovaciones digitales que requieren la construcción de infraestructuras ecosistémicas, más allá de la organización particular, a la cual vincular múltiples actores con diferentes roles en el proceso de creación de innovaciones, producción o entrega de productos y servicios, a los que les es proporcionado, de manera crítica, gobernanza y regulación para desarrollar lógicas legítimas y cursos de acción. Ejemplos de esta forma de infraestructura son las plataformas blockchain y bitcoin (Nambisan, 2016).

- Organizaciones digitales novedosas: Estas son arreglos innovadores de prácticas, estructuras y valores que configuran un núcleo apropiado para cierto contexto institucional. A medida que avanza el estudio de la innovación digital, la teoría institucional invita a investigar cómo se desarrollan las formas nuevas, cómo se difunden y, lo más crítico de este desarrollo y su difusión, cómo ganar legitimidad, generalmente mediante procesos de teorización donde los innovadores presentan argumentos sobre los problemas y asuntos que están resolviendo. Ejemplos de estos arreglos son Airbn y Uber (Strang y Meyer, 1993).

Teóricamente, comprender la naturaleza y fines de la CG referida al uso de la información para la toma de decisiones en diferentes tipos de entidades y organizaciones, representa una forma de poder del conocimiento que ha adquirido presencia al modo de un "eje tranversal" en el que se han borrado las barreras disciplinarias para lograr la consistencia de un mega-explicativo ocupacional.
Por su naturaleza, los conocimientos y la experiencia acumulada por los grupos de trabajo de los profesionales en CG, no son susceptibles de ser tratados en el ámbito de una sola disciplina, por cuya razón, resulta de conveniencia práctica organizarlos como tema transversal que respondan a un proyecto válido dentro del marco social en que se desarrolla toda la educación de los profesionales contables de gestión (Stolkiner, A. 1999, p.2).

Cuando el contador público actúa como un tecnólogo contable de gestión, emplea herramientas conceptuales adoptadas desde distintas disciplinas que aplica en su labor cotidiana, pero que pudiera desconocer en su fundamento o no soler discernir las inconsistencias posibles entre grupos de conceptos provenientes de representaciones comunes a la Contabilidad, la Economía y la Administración.

Los conocimientos aportados por la ciencia económica a la CG se circunscriben al paradigma de la economía neoclásica centrado en la relación con los costes y beneficios de la búsqueda de una mínima satisfacción, sujeta quizás a paulatinos ajustes según las conveniencias del inversor. Por su parte, los conocimientos entregados por la ciencia administrativa se enfocan en el paradigma de la gestión por procesos, que consiste en:

$$
\begin{aligned}
& \text { una forma de conducir efectivamente las activi- } \\
& \text { dades, interrelaciones y recursos de una orga- } \\
& \text { nización, concentrándose en el valor agregado } \\
& \text { para el cliente y las partes interesadas y nace } \\
& \text { de la necesidad de hacer organizaciones más } \\
& \text { eficientes y eficaces que tengan la capacidad de } \\
& \text { adaptarse al entorno cambiante (López, 2008). }
\end{aligned}
$$

Con relación a su enfoque disciplinar, la utilización de la CG implica una transición desde un paradigma de contabilidad normativa tensionado entre diversos intereses usuarios en búsqueda de soluciones contables específicas, hacia una orientación intelectual procedente de un paradigma de la contabilidad positiva, cuyo propósito es obtener una explicación del hecho contable inmerso en la interacción del flujo de los recursos, la financiación y la inversión relacionadas que promueve la eficiencia de las decisiones financieras y no financieras que se espera irá a tomar la gerencia.

El alcance científico de la contabilidad de gestión se centra en el paradigma de la "perspectiva de la contabilidad como información”, propuesto por Christensen y Feltham (2003) para resaltar el papel de la información que afecta las creencias del decisor sobre la consecuencia de sus acciones.

En el marco social en que se desarrolla la educación del profesional contable, los resultados de la investigación "El papel de la contabilidad de gestión en la formación de los contadores públicos-caso Región Caribe colombiano" (Pérez-Grau, et al. 2018) patrocinada por ASFACOP, además de 
proponer que el componente de contabilidad de gestión deba ser integrado e interiorizado por los programas de contaduría pública, se termina allí, planteando un desarrollo curricular del componente de contabilidad de gestión en donde se enfatiza sobre los siguientes temas o materias:

- Organización administrativa (organigramas y descripción de cargos)

- Costos (ABC o el estándar)

- Presupuestos (ABB o el flexible)

- Nómina y factor prestacional

- Control de gestión (BSC)

- Control Interno

- Sistema integrado de información (ERP)Software contable (procedimientos de: compras y ventas, clientes, proveedores, activos, personal, inventarios y logística)

De su lado, en este trabajo los ejes transversales son constructos compuestos por líneas teóricas que atraviesan, vinculan y conectan diversos planteamientos no sólo interdisciplinares, sino transdisciplinar del conocimiento $y$, por tanto, favorecen una visión global o de conjunto de la práctica profesional. La CG constituye un "eje transversal” integrador de contenidos que resuelve problemas concretos que surgen de los núcleos problematizadores en distintas escalas de complejidad y que alcanza un correcto nivel de definición de los diferentes aspectos temáticos: organizacionales, contables, administrativos, económicos, de auditoría, jurídicos, societarios, impositivos, entre otros, todos ellos dentro de un ambiente informatizado.

La Tabla 2 ilustra unas ideas sobre el modo del cómo las líneas de conocimiento definen la deontología de la CG estando soportadas por núcleos problematizadores u objetivadores de estudios caracterizados por seguir procesos de investigación integradores de las diversas materias relacionadas con el contexto de las empresas y los entes sociales.

Es válido aludir aquí, que:

en materia educativa, el objetivo no se centra en resolver los problemas sino en que éste sea utilizado como base para identificar los temas y materias de aprendizaje para su estudio de manera independiente o grupal, es decir, el problema sirve como detonador para que los estudiantes cubran los objetivos de aprendizaje de los cursos, así los conocimientos son introducidos en directa relación con el problema y no de manera aislada o fragmentada (Guevara, 2010, p. |45-|46).

La CG construye conocimientos de interés social a través de la investigación empírica surgidas desde los núcleos pro- blematizadores conducente a la integración de los resultados que aportan los métodos de la modelización y la simulación computacionales, con las teorías sociales discursivas.

Los modelos en perspectiva estática sirven a los contadores para la elaboración de informes reglamentados sobre materias relacionadas con las inversiones en equipos y la formulación de políticas generales y planeación del largo plazo, la escogencia de las líneas de producción que deban ser enfatizadas o no, sus costos, la asignación de precios a los productos o servicios, los costos del mercadeo, y los gastos relacionados con los programas de capacitación. Los modelos en perspectiva dinámica y entre ellos la simulación, son utilizados por los contadores para la planeación y el control de los costos y las operaciones y la reducción de los riesgos asociados con la toma de decisiones y acciones acontecidas en el pasado.

Modelizar un fenómeno social para luego desarrollar experimentos virtuales simulándolo, implica probar ciertas hipótesis razonables acerca de las formas de comportamiento y modos de interacción de los agentes involucrados en determinado cambio social. Una vez codificadas las hipótesis como un programa informático simulable, el investigador puede controlar la ejecución del modelo, manteniendo constante ciertos parámetros con fines exploratorios. Los parámetros definen el experimento virtual puesto a prueba y requieren ser calibrados con datos empíricos siguiendo metodologías cualitativas y cuantitativas (Concari, 200I, p.82).

No hay modelos genéricos, sino que los modelos y simulaciones traducen la realidad problematizada a ciertas ideas teóricas y escala de valores que el modelizador tiene como parte del sistema de referencia. Todo modelo de simulación implica entonces un modelo mental previo, es decir, un modelo nunca será neutral, sino que presupone un sujeto de conocimiento integrado a un determinado contexto histórico-cultural en el que ejerce un rol en función de ciertos intereses y objetivos. En estas condiciones, el modelo (M) configura un instrumento científico intermediario entre el sujeto $(S)$ y el objeto $(\mathrm{O})$, entre cuya relación triádica, la modelización adquiere el carácter de una estrategia para abordar la complejidad social.

Es de esta manera como el eje transversal de la CG puede ser formalizado y expresado como un meta-modelo (Rodríguez y Roggero, 20 I3, p. I I) con sus conceptos, propiedades y relaciones, que puede ser empleado para modelizar distintos casos de estudio, delimitados con el sistema de referencia construido por y para su campo de acción, capaz de producir suficiente evidencia cualitativa y cuantitativa para parametrizar el modelo, calibrarlo y validarlo $y$, finalmente, evolucionar hacia un dialogo entre las ciencias computacio- 
Tabla 2.

Coordinación y orientación posible de cada línea de la contabilidad de Gestión

\begin{tabular}{|c|c|c|c|}
\hline LÍNEAS & CONCEPTUALIZACIÓN & NÚCLEOS & MATERIAS \\
\hline $\begin{array}{llr}\text { La construcción } & \text { de } & \text { arreglos } \\
\text { institucionales } & \text { de } & \text { base } \\
\text { tecnológica } & & \end{array}$ & $\begin{array}{l}\text { Los argumentos de la innovación } \\
\text { derivados del análisis de problemas y } \\
\text { asuntos por resolver }\end{array}$ & $\begin{array}{l}\text { La distribución y utilización de } \\
\text { la información (subsistema } \\
\text { tecnológico) }\end{array}$ & $\begin{array}{l}\text { Sistema integrado de } \\
\text { información y }\end{array}$ \\
\hline \multirow{2}{*}{$\begin{array}{l}\text { El conocimiento acerca del valor } \\
\text { y naturaleza del resultado de la } \\
\text { empresa durante cierto periodo. }\end{array}$} & \multirow{2}{*}{$\begin{array}{l}\text { El papel de la contabilidad de gestión } \\
\text { como el ejercicio de un socio estratégico } \\
\text { para la organización }\end{array}$} & $\begin{array}{l}\text { Los recursos disponibles (el } \\
\text { subsistema organizativo) }\end{array}$ & $\begin{array}{l}\text { O r g a } n \text { i z a c i ó } n \\
\text { administrativa }\end{array}$ \\
\hline & & $\begin{array}{l}\text { La organización del sistema } \\
\text { productivo (el subsistema de } \\
\text { control }\end{array}$ & $\begin{array}{l}\text { Nómina y } \\
\text { prestacional }\end{array}$ \\
\hline
\end{tabular}

La gestión del desempeño de la organización.

La medición del nivel de contribución de cada una de las actividades que son realizadas

La utilización de los medios disponibles, con la intención de mejorar los procesos de trabajo (subsistema de auditoría de procesos).

La asignación de responsabilidades (subsistema Control de gestión

El desarrollo de la práctica de la toma de decisiones por parte de los gerentes.

Las revisiones para asegurar la aproximación entre la planeación y la obtención de resultados.
Los procedimientos de elaboración presupuestal y del análisis de las desviaciones, tipificándola como un instrumento de control del proceso
La adopción de los marcos y prácticas para identificar, medir, gestionar y reportar los riesgos atentatorios del logro de los objetivos de la organización del tablero de mando).

La optimización de la capacidad existente (subsistema de Planeación estratégica planeación)

El aprovisionamiento de

los factores corrientes de la Costos producción (subsistema de costos)

El análisis y la relación de causalidad en las desviaciones contables (subsistema presupuestal)

\section{Control interno}

Cos

Presupuestos nales y las ciencias sociales, para abordar la complejidad de la contabilidad como ciencia social.

\section{Conclusión}

Una conclusión obvia a la que conduce la observación de los procesos de trabajo y el análisis de la naturaleza de los núcleos problematizadores que soportan la práctica y el estudio de la CG, es que la adopción creciente de las tecnologías de información en los procesos de trabajo de las organizaciones ha hecho que el profesional contable sea cada vez menos técnico y más involucrado en tareas de dirección. De aquí, se desprende la otra conclusión acerca de que al nuevo contador corresponde ahora pensar más en las incidencias económicas de la toma de decisiones que se toman en la gerencia y en sus propias habilidades sobre sistemas informáticos para mantener la simbiosis contable-adminis-
Las otras conclusiones, más próximas al ejercicio reflexivo asumido en este trabajo, es que la CG tiene un gran potencial de méritos para posicionarse como una tecnología contable, tal como han sido reconocidas la contabilidad ambiental, la contabilidad forense, la contabilidad nacional, entre otras. Algunas de las condiciones alcanzadas por esta tecnología contable que apoyan esta concepción, son:

- Es una auténtica praxis social cuyo ejercicio requiere el contacto directo e integrado con las entidades e instituciones donde suceden sus cotidianidades

- Ha logrado mostrar un manejo conceptual del tipo de problemas que aborda

- Se ejerce profesionalmente de manera especializada con base en conocimientos actitudes y habilidades acreditadas y de reglamentaciones y licencias para el ejercicio. 
- Existe un cúmulo de publicaciones de contadores que implica sistematizaciones y reflexión crítica sobre sus propias prácticas

- Tiene acumulada una enorme cantidad de libros, artículos y ensayos sobre sus teorías y métodos

Es también concluyente agregar que, durante el acompasamiento del ineludible proceso de consolidación de la CG como disciplina tecnológica, la comunidad contable, gremial y empresarial deberá:

- Armonizar esfuerzos para la orquestación de sus diversas innovaciones digitales

- Promover la determinación de las reglas de conducta óptima para las diferentes situaciones de decisión empresarial, mediante razonamientos hipotético-deductivos.

- Formalizar la realización continua de estudios diversos sobre teoría y práctica de la CG.

Asimismo, para que el proceso de maduración de la CG como tecnología científica contable se traduzca en una instanciación legítima se hace necesario fortalecer el vínculo universidad-empresa y dotar a los institutos y centros de consultoría contable que atienden la actualización de los egresados en ejercicio profesional y a las universidades que ofrecen planes de estudios de contaduría pública, de los avances tecnológicos y científicos de la ciencia de la contabilidad.

\section{Conflicto de intereses}

El autor declara no tener ningún conflicto de intereses.

\section{Referencias bibliográficas}

I. CHRISTENSEN, Peter; FELTHAM, Gerald. Economics of accounting: Vol I. Information in markets. NY: Springer, Series in accounting scholarship, 2003. https://doi.org//0.1007/978-|-46/5-||33-5

2. CIMA-AICPA. Global Management Accounting Principles. London: CGMA Global Management Accounting Principles. 2017. p. 56. Disponible en: https://www.cgma.org/content/dam/cgma/resources/reports/downloadabledocuments/global-management-accounting-principles.pdf

3. CONCARI, Sonia. B., Las teorías y modelos en la explicación científica. En: Ciencia y Educacao. 200I. vol 7, no. I. p. 85-94. Disponible en: http://www.scielo.br/pdf/ciedu/v7nl/06.pdf

4. DIMAGGIO, Paul y POWELL Walter. The iron cage revisited: Institutional isomorphism and collective racionality in organizational fields. In:American Sociological Review. Abril 1983. vol. 48, $n^{\circ}$ 2. p. I47-I60. Disponible en: https://www.uio.no/studier/emner/matnat/ifi/INF9200/ vl0/readings/papers/DeMaggio.pdf
5. GUEVARA, Gabriela.Aprendizaje basado en problemas como técnica didáctica para la enseñanza del tema de la recursividad. En: Intersedes: Revista de las Sedes Regionales. 2010. vol. XI, no. 20. p. I42-167. Disponible en: https://www.redalyc/org/pdf/666/666/9992009.pdf

6. HESFORD, James. W. y POTTER, Gordon. Accounting research in the cornell quarterly: a review with suggestions for future research. In: Cornell Hospitaliaty Quaterly. 2010. vol. 5I, no. 4. p. 502-5I2. doi:10.1 I77/1938965510375030. Disponible en: https://scholarship. sha.cornell.edu/articles/788/

7. HININGS, Bob., GEGENHUBER, Thomas.y GREENWOOD, Royston. Digital innovation and transformation: an institutional perspective. In: Information and Organization. March 2018. vol. 28, no. I. p. 52-6I. https://doi.org/10.1016/j.infoandorg.2018.02.004 Disponible en: http:// isiarticles.com/bundles/Article/pre/pdf/84492.pdf

8. IMA. Managerial costing conceptual framework. NJ: IMA, Managerial Costing Conceptual Framework Task Force, 20I4. Disponible en: https://www.researchgate.net/profile/Charles Thomas5/publication/259359074_Conceptual_Framework_for_Managerial_Costing_Report_of_the_IMA_Managerial_Costing_Conceptual_Framework_Task_Force/links/0deec52b305 I78f7ba000000/Conceptual-Framework-for-Managerial-Costing-Report-of-the-IMA-Managerial-Costing-Conceptual-Framework-Task-Force.pdf

9. KING, lan. New set of accounting principles can help drive sustainable succes. London: Financial Times. Opinion Letter, 23 October 2014. Disponible en: https:/www.ft.com/content/ 0b595a98-59fa-I le4-877I-00I44feab7de

10. LOEBBECKE, Claudia y PICOT, Arnold. Reflections on societal and business model transformation arising from digitization and big data analytics: a research agenda. In: Journal of strategic information systems. September 20I5. vol.24, no. 3. p. I49-I57. Disponible en: $\underline{\text { http:// }}$ iranarze.ir/wp-content/uploads/2016/09/5252-English.pdf

II. LÓPEZ, Francisco. El enfoque de gestión por procesos y el diseño organizacional: El Caso Antioqueño. Marco Teórico. Maestría en Administración. Medellín. EAFIT. 2008. p. 106. Disponible en: https://repository.eafit.edu.co/handle//0784/4496

12. LUNKES, Rogerio; RIPOLL, Vicente y SILVA Fabricia. Estudio de las publicaciones sobre Contabilidad de Gestión de Brasil y España. En: Cont-Fin, USP. 2012. vol. 24, no. 6I. p. II-26. Disponible en: http:// www.scielo.br/pdf/rcf/v24n6l/v24n6la03.pdf

13. MEYER John. W. y ROWAN Brian. Institutionalized organizations: formal structure as myth and ceremony. In: The American journal of sociology, 1977, vol. 83. p. 340-363. Disponible en: https://www.jstor. org/stable/2778293?seq=I\#page_scan_tab_contents

14. NAMBISAN, Satish. Digital entrepreneurship: toward a digital technology perspective of entrepreunership. Entrepreunership: In: Theory and practice. 2016. vol. 4I4. p. I-27. Disponible en: https://www.effectuation.org/wp-content/uploads/2017/06/Nambisan-2016-Entrepreneurship_Theory_and_Practice-I.pdf

15. PEÑAlOZA, Mariana. P. Teoría de las decisiones. En: Perspectiva. 2010, vol. 25. p. 227-240. Disponible en: https://www.redalyc.org/ pdf/4259/4259424540/2.pdf 
16. PÉREZ-GRAU, Samuel; MONSALVE, Magda; RUZ, Alexander; MAESTRE, Genner; ZABALETA, Milena; CABANA, José; TAPIA, Diomedes;TRIANA, Laureano; RAMÍREZ, Sergio y RíOS, Luis. El papel de la contabilidad de Gestión en la formación de los contadores públicos. Barranquilla: Ediciones Simón Bolívar, 2017. 105 p. Disponible en: https://bonga.unisimon.edu.co/bitstream/handle/20.500.12442/26/3/ Preliminares.pdf? sequence $=4 \&$ is Allowed $=y$

17. PÉREZ-GRAU, Samuel y ZABALETA, Milena. ERP: El sistema de planificación de los recursos de la empresa. Barranquilla: Ediciones Universidad Simón Bolívar. 2017. 123p. Disponible en: http://bonga. unisimon.edu.co/handle/20.500.12442//557? show=full

18. PÉREZ-GRAU, Samuel. Los programas paradigmáticos de la contabilidad siglo XXI. En: Entramado. 20I4, vol. I0, no. I. p. I26-I48. Disponible en: https://revistas.unilibre.edu.co/index.php/entramado/article/ view/3484/2875

19. RODRÍGUEZ ZOYA, Leonardo y ROGGERO, Pascal. La modelización y simulación computacional como metodología de investigación social. En: Polis. 2014, vol. 39, no. 3. p. 25-50 Disponible en: https:// journals.openedition.org/polis//0568

20. STOLKINER Alicia. La interdisciplina entre la epistemología y la práctica. En: El Campo Psi. 1999. vol. 10, no. 3. p. 25-34. Disponible en: http://www.psi.uba.ar/academica/carrerasdegrado/psicologia/sitios catedras/obligatorias/066_salud2/material/unidadI/subunidad_I_3/ stolkiner_interdisciplina_salud_mental.pdf

2I. STRANG, David y MEYER, John. Institutional conditions of diffusion. In: Theory and Society. 1993 vol. 22, no. 2. p. 487-5 II. DOI: 10.1007/BF00993595. Disponible en: https://link.springer.com/article/l0.1007\%2FBF00993595

22. THORNTON, Patricia. H.; OCASIO, William y LOUNSBURY, Michael. (20I2). The institutional logics perspective: a new approach to culture, structure and processes. Oxford: Oxford University Press. Disponible en: https://www.cairn.info/revue-management-2012-5-page-583.htm

23. WILLIAMSON, Oliver. E. The new institutional economics: taking stock, looking ahead. In: Journal of economics literatura. 2000, vol. 38, no.3. p. 595-6I3. DOI: 10.1257/jel.38.3.595. Disponible en: https:// www.aeaweb.org/articles?id=10.1257/jel.38.3.595 